\title{
XLIX. Decades novarum specierum florae katangensis. XII-XIV.
}

Von E. De Wildeman.

(Originaldiagnosen.)

LVII. Aneilema R. Bz. (Commelinaceae).

\section{Aneilema angustifolium De Wild., nov. spec.}

Ramis plus minus ramosis, usque $20 \mathrm{~cm}$ longis, breviter et sparse velutinis; foliis vaginantibus, vagina usque $7 \mathrm{~mm}$ longa, lamina ellipticolanceolata, $15-20 \mathrm{~mm}$ longa, et $7 \mathrm{~mm}$ lata, glabra vel sparse velutina, nervis I utrinque $2-3$; inflorescentiis paniculatis terminalibus usque $3 \mathrm{~cm}$ longis et $15 \mathrm{~mm}$ latis, pedunculatis, pedunculo $13 \mathrm{~mm}$ longo, bracteis circ. $2 \mathrm{~mm}$ longis, floribus luteis; capsula sparse velutina, 5-6 mm longa et circ. $3 \mathrm{~mm}$ lata, apice rotundato-cuneata.

Ober-Katanga: Sangatele, sept. 1911 (Ad. Hock).

Obs. - Verwandt mit $A$. Johnstonii Schum., verschieden durch kleinere Blätter und kleinere Infloreszenzen.

112. Aneilema erectum De Wild., nov. spec.

Ramis erectis plus minus ramosis, usque $45 \mathrm{~cm}$ altis; foliis basilaribus reductis; medianis lamina usque $15 \mathrm{~mm}$ longa; superioribus vagina paullo inflata, circ. $10 \mathrm{~cm}$ longa, lamina lanceolata $3-8 \mathrm{~cm}$ longa et $14 \mathrm{~mm}$ lata, supra et intra plus minus velutina, apice cuneato-acuta, basi cuneata, nervis longitudinalibus I utrinque circ. 5 ; inflorescentiis paniculato-ramosis, usque $5 \mathrm{~cm}$ longis et $3 \mathrm{~cm}$ latis, pedunculatis, pedunculo velutino-hirsuto $\mathbf{j} \mathrm{cm}$ longo, versus apicem folio bracteiformi $1,5 \mathrm{~cm}$ longo minuto, ramis densis, pedicellis velutinis, bracteis circ. $3 \mathrm{~mm}$ longis. Capsula circ. $7 \mathrm{~mm}$ longa et $4 \mathrm{~mm}$ lata, apiçe plus minus cuneata.

Ober-Katanga: Élisabethville, octobre 1911 (Ad. Hock).

Obs. - Scheint mit $A$. katangensis De Wild. verwandt zu sein. aber sehr bequem verschieden durch die Blattform.

113. Aneilema Florenti De Wild., nov. spec.

Ramis plus minus erecto-decumbentibus, usque $35 \mathrm{~cm}$ altis, velutinoscabris; foliis lanceolatis, lamina supra et infra glabra, apice acuta. usque $10 \mathrm{~cm}$ longa et $18 \mathrm{~mm}$ lata; nervis longitudinalibus I utrinque circ. 5; paniculis dense ramosis terminalibus usque $3 \mathrm{~cm}$ longis et $2,5 \mathrm{~cm}$ latis, pedunculatis, pedunculo circ. $4 \mathrm{~cm}$ longo et versus medio folio bracteiformi reducto, plus minus papyraceo, munito, ramis velutinis, bracteis circ. $2 \mathrm{~mm}$ longis, pedicellis gracilibus post anthesin usque $5-$ $6 \mathrm{~mm}$ longis, sepalis glabris circ. $4 \mathrm{~mm}$ longis, obtusis, petalis luteis, unguiculatis, circ. $5 \mathrm{~mm}$ longis; capsula ellipsoidea, sparse velutinoscabra, apice late cuneato-nbtusa, $8-9 \mathrm{~mm}$ longa et circ. $5 \mathrm{~mm}$ lata, 2-loculata, loculis 2 -seminatis, seminibus $3 \mathrm{~mm}$ circ. longis et $2 \mathrm{~mm}$ latis, dorso carinatis.

Repertorium specierum novarum. XII. (24. VII. 1913.) 
Ober-Katanga: Welgelegen, 1912 (leg. Florent, coll. Homblé, no. 640$)$.

Obs. - Diese Art gehört zu der Gruppe: Lamprodithyros und ist mit A. Homblei De Wild. und A. Johnstonii Schum. verwandt; sie unterscheidet sich von diesen Arten durch die Blattform, durch das Indumentum und durch die am Stiel der Infloreszenz merkwürdige Braktee.

114. Aneilema Hockii De Wild., nov. spec.

Ramis erectis plus minus ramosis, usque $40 \mathrm{~cm}$ longis, velutinis; foliis lamina usque $15 \mathrm{~cm}$ longa et $18 \mathrm{~mm}$ lata, apice plus minus acuta, foliis superioribus reductis, inflorescentiis terminalibus plus minus longe pedunculatis, pedunculo $4-6 \mathrm{~cm}$ circ. longo, rachide velutino, ramis bracteatis, bracteis vaginantibus usque $3 \mathrm{~mm}$ longis $3-5 \mathrm{~mm}$ distantibus; floribus pedicellatis, pedicello gracili, usque $7 \mathrm{~mm}$ circ. longo post anthesin accrescenti, lobis calycis usque $11 \mathrm{~mm}$ longis et $3 \mathrm{~mm}$ latis; cnpsula $7-10 \mathrm{~mm}$ longa, glabra, plus minus angulata apice truncata, biloculato, loculis biseminatis.

Ober-Katanga: Élisabethville, 1911 (Ad. Hock. - Sur termitière).

Obs. - Diese Art gehört zu der Gruppe Dicarpellaria und die UnterGruppe Amelina; sie kommt in die Verwandtschaft von A. aequinoctiale Kunth und A. nyasense C. B. Clarke, doch ist mit dieser letzten speziell verwandt, hat wie diese wenige Samen; sie unterscheidet sich von $A$. myasense durch längere und schmälere Blätter.

115. Aneilema Homblei De Wild., nov. spec.

Ramis plus minus ramosis usque $30 \mathrm{~cm}$ longis, glabris vel sparse et breviter velutinis; foliis ellipticis vel ovato-lanceolatis, $4-10 \mathrm{~cm}$ longis et $1-3 \mathrm{~cm}$ latis, breviter velutinis, margine ciliatis, nervis lateralibus 1 utrinque circ. 5; paniculis ramosis densis, terminalibus usque $4 \mathrm{~cm}$ circ. longis et $3 \mathrm{~cm}$ latis, pedunculatis, pedunculo usque $5 \mathrm{~cm}$ longo, bracteis densis circ. $2 \mathrm{~mm}$ longis, pedicello gracili post anthesin accrescenti et usque $5 \mathrm{~mm}$ longo; sepalis glabris, circ. $3 \mathrm{~mm}$ longis apico obtusis, petalis luteis, unguiculatis, usque $5 \mathrm{~mm}$ longis et $4 \mathrm{~mm}$ latis. capsula subglabra, ellipsoidea, apice obtusa, leviter apiculata $6 \mathrm{~mm}$ circ. longa et circ. $5 \mathrm{~mm}$ lata biloculata; loculis 2 -seminatis; seminibus circ. $2 \mathrm{~mm}$ longis et latis.

Ober-Katanga: Shisangwe, janvier 1912 (Homblé, no. 111).

Obs. - Diese Art der Gruppe Dicarpellaria-Lanprodithyros, kommt in die Nähe von A. angolense C. B. Clarke und A. Johnstonii Schum., durch die Infloreszenz ist sie mit der letzlen stark verwandt, unterscheidet sich durch grössere Blätter und kondensierte Blütenstände.

116. Aneilema katangensis De Wild., nov. spec.

Ramis erectis plus minus ramosis usque $70 \mathrm{~cm}$ longis; foliis basilaribus reductis, glabris vel dorso sparse velutinis, medianis usque $6 \mathrm{~cm}$ longis et $1,5 \mathrm{~cm}$ latis, superioribus vaginatis, vagina circ. $12 \mathrm{~mm}$ longa, lamina ovato-elongata, basi plus minus cordata, $8-11 \mathrm{~cm}$ longa et $22--$ $30 \mathrm{~mm}$ lata, nervis lateralibus I utrinque $4-5$, inflorescentiis $4,5 \mathrm{~cm}$ longis et $4 \mathrm{~cm}$ latis, pedunculatis, pedunculo circ. $6 \mathrm{~cm}$ longo medio 
folio bracteiformi munito; rachide velutino, bracteis circ. $3 \mathrm{~mm}$ longis; pedicellis gracilibus post anthesin accrescentibus usque $.5 \mathrm{~mm}$ longis; floribus luteis, sepalis glabris, apice obtusis circ. $4 \mathrm{~mm}$ longis; capsula ellipsoidea sparse pilosa, apice rotundato-cuneata circ. $7 \mathrm{~mm}$ longa et 4-5 mm lata; biloculata, loculis biseminatis.

Ober-Katanga: Shilongo, septembre 1911 (Ad. Hock).

Obs. - Wie $A$. Homblei und $A$. Florenti, ist $A$. katangense mit $A$. Johnstonii K. Schum. verwandt, sie ist doch durch die Blattlorm sehr gut verschieden.

117. Aneilema Ringoeti Do Wild., nov. spec.

Ramis erectis, velutinis, usque $25 \mathrm{~cm}$ altis, radicis fibrosis; folis vaginatis, vagina plus minus inflata usque $11 \mathrm{~mm}$ longa et $6-7 \mathrm{~mm}$ crassa, lamina breviter et sparse velutina, elliptico-oblonga, 1,3-5 cm longa et 6-14 mm lata, apice plus minus longe cuneata, nervis lateralibus I utrinque circ. 4; inflorescentiis. terminalibus $14-20 \mathrm{~mm}$ latis et $18 \mathrm{~mm}$ latis, pedunculatis, pedunculo breviter tomentoso, $15-20 \mathrm{~mm}$ longo; floribus coeruleis; capsulis oblongis circ. $4,5 \mathrm{~mm}$ longis et $3,3 \mathrm{~mm}$ latis.

Ober-Katanga: Shinsenda, mars 1912 (Ringoet, coll. Homblé, no. 545$)$.

Obs. - Durch diese Merkmale ist die neue Art in die Verwandtschaft von A. Weluitschii C, B. Clarke (cf. Fl. of trop. Afr., VIII, p. 76) zu rechnen.

Die Gattung Aneilema ist ganz neu monographisch zu bearbeiten, es fehlt uns noch zu viel Material, um diese Arbeit zu übernehmen. Wir hoffen aber, bald von Zentral-Afrika neues Material zu bekommen.

\section{Commelina L. (Commelinaceae).}

118. Commelina Bequaerti De Wild., nov. spec.

Ramis plus minus repentibus, usque $30 \mathrm{~cm}$ longis, internodiis usque $6,5 \mathrm{~cm}$ longis; foliis lanceolatis, vaginantibus, vagina $1 \mathrm{~cm}$ circ. longa velutina, lamina $2,5-10 \mathrm{~cm}$ longa et $6,5 \mathrm{~mm}$ lata, supra et infra velutina, apice acuta; inflorescentiis pedunculatis, pedunculo velutino usque $3,5 \mathrm{~cm}$ longo, spatha usque $19 \mathrm{~mm}$ longa, ovata, basi rotundata, apice acuta et $12 \mathrm{~mm}$ lata, breviter hispida, striata, margine basi connata; petalis coeruleis usque $8 \mathrm{~mm}$ longis, unguiculatis et $6 \mathrm{~mm}$ latis; capsula circ. $4,5 \mathrm{~mm}$ longa, 5 seminata, seminibus globulosis, laevis circ. $1,5 \mathrm{~mm}$ diam.

Ober-Katanga: Élisabethville, 28 mars 1912 (J. Bequaert, no. 293).

Obs. - Verwandt mit C. zambesica C. B. Clarke (Fl. of trop. Afr., VIII, p. 43).

119. Commelina Homblei De Wild, nov. spec.

Ramis repentibus usque $15 \mathrm{~cm}$ longis, foliis vaginantibus, vagina brevi usque $6 \mathrm{~mm}$ longa, velutina, internodiis usque $3 \mathrm{~cm}$ longis, lamina lanceolata usque $7,5 \mathrm{~cm}$ longa et $12,5 \mathrm{~mm}$ lata, velutino-hirsuta, inflores- 
centiis pedunculatis, pedunculo solitari usque $3 \mathrm{~cm}$ longo, spatha velutino-hirsuta circ. $2 \mathrm{~cm}$ longa, apice acuta, basi rotundata, margine basi connata; petalis coeruleis circ. $7 \mathrm{~mm}$ longis; capsula $4,5 \mathrm{~mm}$ circ. longa $4-5$ seminata.

Ober-Katanga: Élisabethville, février 1912 (Homblé. no. 124).

Obs. - Diese Art ist wie C. Bequaerti mit C. zambesica C. B. Clarke verwandt; ihre Blätter sind zweimal so gross wie die von $C$. Bequaerti.

\section{Chironia L. (Gentianaceae).}

120. Chironia katangensis De Wild., nov. spec.

Herbacea, erecta usque $50 \mathrm{~cm}$ alta; caulis cylindricis parce ramosis, foliis lineari-lanceolatis, $15-27 \mathrm{~mm}$ longis et $1,5-3 \mathrm{~mm}$ latis, oppositis, internodiis usque $9,5 \mathrm{~cm}$ longis; floribus $1-6$, pedicellatis, pedicello usque $2,8 \mathrm{~cm}$ longo, sepalis linearibus, circ. $13 \mathrm{~mm}$ longis et paulo ultra $1 \mathrm{~mm}$ lata, acutis, carinatis; corolla rosea vel rubra, tubo $5-6 \mathrm{~mm}$ longo, lobis usque $19 \mathrm{~mm}$ longis lanceolato-icutis et circ. $5 \mathrm{~mm}$ latis; staminibus exsertis, filamentis brevibus, antheris $6--6.5 \mathrm{~mm}$ longis basi cordatis; stylo corollae lobis subaequilongo apice breviter inflato.

Ober-Katanga: Elisabethville, mars 1912 (Homblé, no, 280) et 4 avril 1912 (J. Bequaert, no. 319)

Obs. - Diese Art ist mit C. transvaalensis Gilg (ef. Fl. of trop. Afr., IV, 1, p. 555) nahe verwandt.

\section{L.X. Asparagus L. (Liliaceae).}

\section{Asparagus Bequaerti De Wild, nov. spec.}

Ramis erectis, ramosis, glabris, ramis divergentibus, circ. $20 \mathrm{~cm}$ longis; folijs calcaratis in spinas recurvatas productis; cladodia pauci numerosa ad apices ramulorum solitaria, setacea apice leviter recurvata. 10-12 mm longa; floribus axillaribus, solitaribus vel geminatis, pedicellatis, pedicello usque $6 \mathrm{~mm}$ longo versus basin articulato, perianthii lobis $4 \mathrm{~mm}$ circ. longis.

Ober-Katanga: Sankisia, 21 septembre 1911 (J. Bequaert, no. 205).

Obs. - Verwandt mit A. equisetoides Welw. und A. nudicaulis Baker, unterscheidet sich von der ersten Art durch längere Cladodien, von der zweiten durch die zu zwei vereinigten Blüten und durch die Artikulation der Blütenstiele.

122. Asparagus Homblei De Wild., nov. spec.

Ramis erectis, plus minus ramosis, ramis usque $60 \mathrm{~cm}$ longis, plus minus angulosis, glabris; foliis calcaratis in spinas $5 \mathrm{~mm}$ circ. longas paullo recurvatas productis; cladodia numerosa usque 10 na, plus minus recurvata, usque $5 \mathrm{~cm}$ longa, filiformia, acuta; floribus ....; baccae globulosae, rubrae, $7-8 \mathrm{~mm}$ latae, pedicellatae, pedicello $5-6 \mathrm{~mm}$ longo $1,5 \mathrm{~mm}$ supra basi articulato.

Ober-Katanga: Février 1912 (Homblé, no. 172. - In termitière).

Obs. - Diese Art kommt in die Nähe von A. mozambicus, sio hat längere Cladoden, und diese sind zu mehreren in einem Fascikel vereinigt. 
113. Asparagus striatus De Wild., nov. spec.

Ramis erectis usque $60 \mathrm{~cm}$ altis longitudinaliter striatis, scabris, ramis striatis, scabris, usque $25 \mathrm{~cm}$ longis; foliis calcaratis in spinas paulo recurvatas usque $9 \mathrm{~mm}$ longas productis; cladodia solitaria, geminata vel $3-4$ fasciculata, subulata vel compressa, acuta, $13 \mathrm{~mm}$ circ. longa et $0,7 \mathrm{~mm}$ lata; floribus axillaribus solitaribus, geminatis vel usque 4. fasciculatis, pedicellatis, pedicello circ. $2 \mathrm{~mm}$ longo supra medium articulato; perianthio $3 \mathrm{~mm}$ circ. longo.

Ober-Katanga: Kakonde, septembre 1911 (Ad, Hock).

Obs. - Diese Pflanze unterscheidet sich von $A$. deflexus, A. Nelsii und $A$. mossambicus durch den stark und scharf gestreiften Stengel; man bann auch andere Verschiedenheiten in der Form und Länge der Cladoden finden.

124. Asparagus Kaessneri De Wild., nov. spec.

Rämis erectis circ. $70 \mathrm{~cm}$ longis, breviter velutinis, plus minus cylindricis, ramis elongatis erectis; foliis calcaratis in spinas apice paullo recurvatas productis; cladodia solitaria vel geminata, raro $3-4$ fasciculata, rigida, subulata, erecta, $8-18 \mathrm{~mm}$ longa; floribus solitaribus vel geminatis, pedicellatis, pedicello usque $9 \mathrm{~mm}$ longo versus basin articulato; perianthio $3-3,5 \mathrm{~mm}$ longo.

Zentral-Afrika-Seengebiet: Vallée de la Rusisi, 22 juillet 1908 (hassner, no. 3178).

Obs, - Diese Art ist mit A. Schweinfurthii Baker verwandt; sie ist erkennbar durch die kurze Behaarung, durch kurze Cladoden und kürzere Blütenstiele, grössere Blüten.

\section{XLXI. Cyanotis Don (Commelinaceae).}

125. Cyanotis minima De Wild., nov. spec.

Planta reducta, ramis repentibus usque $8 \mathrm{~cm}$ longis, radicis fibrosis; caulibus velutinis, plus minus hirsutis, foliis vaginantibus, vagina usque $6 \mathrm{~cm}$ longa, lamina elliptica vel ovato-lanceolata, $12-25 \mathrm{~mm}$ longa et 5-6 mm lata, margine longe ciliata, ramis floriferis non ramosis, inflorescentiis brevibus paullo exsertis, floribus coeruleis, capsula circ. $1,5 \mathrm{~mm}$ lata, plus minus longe velutino-hirsuta.

Ober-Katanga: Shinsenda, 27 février 1912 (Ringoet, coll. Homblé, no. 402).

Obs. - Diese Art scheint mit C. somaliensis C. B. Clarke nahe verwandt zu sein; sie hat doch kleinere Blätter, wir kennen nur fertile Stüche.

\section{Cynometra L. (Jeguminosaceae).}

126. Cynometra Bequaerti De Wild., nov. spec.

Frutex, ramis lignosis; foliis paripennatis, 2 -jugis, rachide $1,4-4 \mathrm{~cm}$ longo, foliolis supra et infra glabris, 5-15 cm longis et $15-46 \mathrm{~mm}$ latis, inaequilateralibus, basi cuneatis, apice acuminatis, emarginatis, nervis lateralibus I utrinque ultra 10, arcuatim anastomosantibus, reticulatio prominenti; inflorescentiis axillaribus solitaribus vel geminatis, pe- 
dunculatis, pedunculo usque $2 \mathrm{~cm}$ circ. longo, tomentoso, versus basim bracteolato, bracteolis linearibus, caducis; calycis tubo $3,5 \mathrm{~mm}$ circ. longo, lobis lanceolatis vel ovato-lanceolatis, refractis usque $9 \mathrm{~mm}$ longis et $1-3,5 \mathrm{~mm}$ latis, petalis erectis subaequalibus, obovato-oblongis, usque $13 \mathrm{~mm}$ longis et $2-3,5 \mathrm{~mm}$ latis; staminum flamentis glabris, basi coalitis, usque $14 \mathrm{~mm}$ longis, antheris circ. $1 \mathrm{~mm}$ longis; ovario dense velutino, stylo glabro.

Ober-Katanga: Entre Ponthierville et Kindu, 29 octobre 1910 (J. Bequaert, no. 53).

\section{Dasystachys Baker (Liliaceae).}

127. Dasystachys Bequaerti De Wild., nov. spec.

Foliis basilaribus linearibus usque $35 \mathrm{~cm}$ longis et $1 \mathrm{~cm}$ latis, glabris, apice margine breviter ciliatis; pedunculo subcylindrico, usque $60 \mathrm{~cm}$ longo, 8 foliato, foliis reductis vaginantibus, apice acutis; floribus breviter pedicellatis, pedicello circ. $1 \mathrm{~mm}$ longo, bracteato, bracteis scariosis usque $6 \mathrm{~mm}$ longis, lanceolatis, acutis, perianthio $5-6 \mathrm{~mm}$ longo, albido. extus glabro, non dorso nigro carinato; staminibus exsertis, antheris luteolis circ. $1.5 \mathrm{~mm}$ longo, stylo exserto.

Ober-Kat anga: Élisabethville, 15 avril 1912 (J. Bequaert, no. 533).

Obs. - Verwandt mit D. papillosa Baker (cf. Fl. of trop. Afr., VII, p. 514), unterscheidet sich durch die Grösse der Blüten und andere Nerkmale, die man aus der Vergleichung der Diagnosen finden kann.

LXIV. Drimia Jacq. (Liliaceae).

128. Drimia Hockii De Wild., nov. spec.

Bulbosa, bulbo globuioso rel ovoideo, usque $3 \mathrm{~cm}$ lato, foliis jgnotis, scapo non maculato, usque $50 \mathrm{~cm}$ longo, racemo $7-20 \mathrm{~cm}$ longo circ. 10-floro, floribus plus minus distantibus, pedicellatis, pedicello usque $6 \mathrm{~cm}$ longo, erecto. basi bracteato, bracteis caducis. linearibus usque $9 \mathrm{~mm}$ longis; perianthio rubeolo, $11-13 \mathrm{~mm}$ longo, tubo plus minus campanulato, 4-5 mm longo, segmentis refractis, linearibus, uni-nerviatis, usque $1,5 \mathrm{~mm}$ latis, staminum filamentis violaceis, antheris circ. $1 \mathrm{~mm}$ longis. stylo breviter exserto.

Ober-Katanga: Élisabethville, septembre 1911 (Ad. Hock); Bukama. 14 juillet 1911 (J. Bequaert, no. 146).

Obs. - Diese Art kommt in die Nähe von D. Coleae Baker (cf. Fl. of trop. Afr., VII, p. 525), ist aber von dieser Pflanze durch das Fehlen von Blättern in der Blütezeit, die Länge des Blïtenstandes usw. verschieden.

\section{Glycine L. (Leguminosaceae).}

129. Glycine moerensis De Wild, nov. spec.

Suffrutex?, ramis basi lignosis, erectis, usque $30-40 \mathrm{~cm}$ altis, longitudinaliter striatis, dense velutinis, pilis plus minus refractis; stipulis lanceolatis, plus minus persistantibus usque $5 \mathrm{~cm}$ longis et $1,5 \mathrm{~cm}$ latis: foliis trifoliolatis, petiolo velutino, usque $2.5 \mathrm{~cm}$ longo, foliolis lateralibus breviter petiolulatis, petiolulo circ. $2-3 \mathrm{~mm}$ longo, ellipticis, la- 
teralibus paullo vel non inaequilateralibus, velutinis, supra et infra subscabris, $4.5-7 \mathrm{~cm}$ longis et $8-25 \mathrm{~mm}$ latis; nervis lateralibus I utrinque circ. 6 - 7 ; inflorescentiis axillaribus vel terminalibus, plus minus longe pedunculatis, pedunculo usque $9,5 \mathrm{~cm}$ longo; floribus albidis, capitulatis, dense bracteolatis, bracteolis linearibus usque $5 \mathrm{~mm}$ longis; caIyce dense velutino, usque $5 \mathrm{~mm}$ longo, vexillo $6 \mathrm{~mm}$ longo, emarginato et $3 \mathrm{~mm}$ lato; ovario velutino.

Ober-Katanga: Kilwa (Moero), novembre 1904 (100 m alt.).

Obs. - Ist mit G. Bequaerti nob. und G. holophylla (Baker) Taub. verwandt; hat aber Blätter mit drei Blättchen.

130. Glycine Ringoeti De Wild,, nov. spec.

Ramis volubilis, polygonalibus, pilosis, pilis brunneis densis, refractis; stipulis caducis; foliis trifoliolatis, petiolatis, petiolo velutino, usque $5, \overline{c m}$ longo; foliolis petiolulatis, stipellatis, stipellis linearibus usque $2,5 \mathrm{~mm}$ longis, petiolo velutino $3-4 \mathrm{~mm}$ longo, lamina longe elliptica, basi rotundata vel subcordata, apice rotundata, apiculata, supra et infra velutina, $4-7,5 \mathrm{~cm}$ longa et $9-20 \mathrm{~mm}$ lata, nervis I utrinque $7-9$; racemis axillaribus, usque $50 \mathrm{~cm}$ longis, laxis, rachide dense velutino; floribus solitaribus, geminatis vel fasciculatis, basi bracteolatis, beacteolis velutinis, ciliatis; pedicello usque $3 \mathrm{~mm}$ longo; calyce velutino, $4 \mathrm{~mm}$ circ. longo bibracteolato, bracteolis ciliatis $2 \mathrm{~mm}$ circ. longis; corolla rubra circ. $5 \mathrm{~mm}$ longo, vexillo $5 \mathrm{~mm}$ longo.

Ober-Katanga: Shinsenda, mars 1912 (Ringoet, coll. Homblé, no. 466).

Obs. - Verwandt mit G. Gilletii nob, aus West-Kongo.

\section{Fadogia Schweinf. (Rubiaceae).}

131. Fadogia Homblei De Wild., nov. spec.

Ramis erectis, usque $50 \mathrm{~cm}$ altis, foliis 4-verticillatis, elliptico-lanceolatis, subsessilibus, basi cuneatis, apice cuneato-acuminatis, supra glabris, infra breviter tomentosis, nervis lateralibus I circ. utrinque $8-9$; stipulis lanceolatis, lobis liberis usque $13 \mathrm{~mm}$ longis; floribus axillaribus fasciculatis, breviter pedicellatis, pedicellis usque $4 \mathrm{~mm}$ longis; calyce glabro, tubo circ. $1.5 \mathrm{~mm}$ longo, lobis lanceolatis, acutis $3 \mathrm{~mm}$ longis; tubo corollae circ. $4 \mathrm{~mm}$ longa, lobis refractis circ. $4 \mathrm{~mm}$ longis, glabris. acutis; staminibus parce exsertis, stylo glabro, stigmato calyptriformi. circ. $1 \mathrm{~mm}$ longo.

Ober-Katanga: Shinsenda, mars 1912 (coll. Homblé, no, 555).

Obs, - In die Verwandtschaft von $F$, agrestis Schweinf., doch durch die Blätter gut verschieden.

132. Fadogia katangensis De Wild., nov. spec.

Planta mediocra, basi lignosa, ramis erectis usque $35 \mathrm{~cm}$ longis. gracilibus non ramosis; foliis 3 -verticillatis, lanceolato-acutis, basi longe cuneatis, plus minus coriaceis, supra sparse pilosis, infra tomentosis: inferioribus circ. $7 \mathrm{~cm}$ longis, medianis $8 \mathrm{~cm}$ longis et $14 \mathrm{~mm}$ latis, margine revolutis, nervis supra impressis, infra prominentibus; stipulis 
vaginantibus, lobis liberis usque $1 \mathrm{~cm}$ longis, margine ciliatis; floribus pedicellatis, pedicello velutino $3 \mathrm{~mm}$ circ. longo et bracteolato; calyce sparse velutino, tubo $2 \mathrm{~mm}$ circ. longo, lobis erectis $1,5 \mathrm{~mm}$ circ. longis; tubo corollae extus sparse velutino, circ. $3 \mathrm{~mm}$ longo, lobis refractis, acutis; staminibus paullo exsertis, stylo exserto, stigmate bilobato, calyptriformi circ. $1 \mathrm{~mm}$ longo.

Ober-Katanga: Élisabethville, septembre 1911 (Ad. Hock).

Obs. - Verwandt mit F. Butayei De Wild. von Kwango (WestKiongo).

133. Fadogia Ringoeti De IVild., nov. spec.

Ramis erectis et plus minus ramosis, usque $80 \mathrm{~cm}$ altis, foliis 3verticillatis, sessilibus, lamina basi rotundata vel lata cuneata, ellipticolanceolata, 4-5,5 cm longa et 7-10 $\mathrm{mm}$ lata, supra et infra glabra, margine sparse pilosa, apice obtuse cuneata; stipulis basi dilatatis usque $1 \mathrm{~mm}$ longi, apice linearibus usque $3 \mathrm{~mm}$ longi; floribus axillaribus, racemis plus minus dichotomibus, rachide usque $9 \mathrm{~mm}$ longo, pedicellis usque $4 \mathrm{~mm}$ longis, glabris; calyce circ. $2 \mathrm{~mm}$ longo, apice truncato, margine undulato; corollae tubo glabro circ. $5 \mathrm{~cm}$ longo, lobis refractis glabris $5 \mathrm{~mm}$ cire. longis, antheris circ. $2 \mathrm{~mm}$ longis, exsertis, stylo exserto, stigmate calyptriformi.

Ober-Katanga: Shinsenda, mars 1912 (leg. Ringoet, coll, Homblé. no. 509).

Obs. - Verwandt mit F. stenophylla Welw. aus Huilla (Angola); sie unterscheidet sich durch die Blattform, grössere Blüten und durch die Infloreszenzen.

\section{Gladiolus L. (Iridaceae).}

134. Gladiolus Corbisieri De Wild., nov. spec.

Bulbus $9-20 \mathrm{~mm}$ diam., bracteis basilaribus $1-2$, scariosis; foliis ช-10, medianis lamina usque $18 \mathrm{~cm}$ longa et $1 \mathrm{~cm}$ lata; spica densa, floribus unilateraliter dispositis, $8-17 \mathrm{~cm}$ longa, $3-8$ flora, spathae valvis lanceolatis, rigidis, imbricatis, plus minus purpureis, usque $42 \mathrm{~mm}$ longis et $12 \mathrm{~mm}$ latis, perianthio purpureo $4 \mathrm{~cm}$ longo, tabo gracili $16 \mathrm{~mm}$ circ. longo, lobo horizontali trilobato apice cucullato obtuso $23 \mathrm{~mm}$ circ. longo et $12-13 \mathrm{~mm}$ lato, lobis lateralibus unguiculatis; antheris linearibus circ. $8-9 \mathrm{~mm}$ longis.

Ober-Katanga: Welgelegen, 1912 (Corbisier, coll. Homblé, no. 602); Shinsenda, mars 1912 (Ringoet, coll. Homblé no. 435 et 518); Mugile W, 23 mai 1908 (Kassner, no. 2996); Shiwele, 19 février 1908 (Kassner, no. 2474).

Obs. - Verwandt mit G. Hanningtoni Baker.

135. Gladiolus Debeersti De Wild., nov, spec.

Caulis erectus circ. 60 , cm altus, vaginis basilaribus $2-3$, foliis liberis 2 , circ. $50 \mathrm{~cm}$ longis, foliis superioribus $2-3$, longe vaginantibus, lamina max. $2 \mathrm{~mm}$ lata; scapo usque $40 \mathrm{~cm}$ longo, inflorescentiis laxis $i-10 \mathrm{~cm}$ longis, floribus $4-5$ unilateraliter dispositis, spathae valvis lanceolatis, usque $2 \mathrm{~cm}$ longis, perianthio violaceo usque $3 \mathrm{~cm}$ longo, 
tubo leviter recurvato, circ. $15 \mathrm{~cm}$ longo, angusti, segmentis subaequilongis, antheris circ. $5 \mathrm{~mm}$ longis.

Ober-Katanga: Tanganika (R. P. Debeerst).

Obs. - Verwandt mit G. zambesiacus Baker (cf. Fl. of trop. Alr., VII, p. 364).

136. Gladiolus katubensis De Wild., nov, spec.

Caulis erectus ultra $80 \mathrm{~cm}$ longus, basi $2-3$ vaginatus, vaginis dorso velutinis; foliis $3-4$ ensiformis, rigidis usque $30 \mathrm{~cm}$ longis et $17 \mathrm{~mm}$ latis, dorso velutinis; spica laxa circ. $50 \mathrm{~cm}$ longa, 7 -flora, spathae valvis viridibus, lanceolatis, apice cuneatis, usque $5 \mathrm{~cm}$ longis; perianthio $7 \mathrm{~cm}$ circ. longo, tubo leviter recurvato usque $6-7 \mathrm{~mm}$ lato, perigonii lobo supremo cucullato; antheris $17 \mathrm{~mm}$ circ. longis.

N.-W.-Rhodesia: Katuba, 30 décembre 1907 (Kassner, no. 2268).

Obs. - Verwandt mit G. velutinus nob.

- 137. Gladiolus paucifiorus De Wild., nov. spec.

Bulbus circ. $3 \mathrm{~cm}$ latus, caulis erectus ultra $75 \mathrm{~cm}$ longus, basi $3-4$ foliis reductis munitus, foliis liberis circ. 3 , glabris, longe vaginantibus, pars libera $15 \mathrm{~cm}$ longa et $15 \mathrm{~mm}$ lata, nervo mediani excentrici; inflorescentiis $29 \mathrm{~cm}$ longis, laxis, $3-4$ floris unilateraliter recurvatis; spathae valvis rubro-viridibus, lanceolatis usque $5,3 \mathrm{~cm}$ longis; perianthio luteolo, usque $8 \mathrm{~cm}$ longo, tubo erecto apice leviter recurvato circ. $4 \mathrm{~mm}$ lato, perigonii lobo supremo cucullato; filamentis staminum recurvatis.

Ober-Katanga: Bugege, septembre 1911; Katanga, octobre 1911 et Shinsenda, décembre 1911 (Ad. Hock).

Obs. - Verwandt mit A. sulphureus Baker (Fl. of trop. Afr., VII, p. 370$)$.

138. Gladiolus velutinus De Wild., nov. spec.

Caulis erectus usque $70 \mathrm{~cm}$ longus basi foliis reductis vaginantibus munitus, vaginis dorso velutinis, foliis 4 , rigidis, lamina usque $30 \mathrm{~cm}$ longa et $10-19 \mathrm{~mm}$ lata, dense velutina: spica laxa $25 \mathrm{~cm}$ circ. longa, 5-7 flora; spathae valvis viridibus, lanceolatis, longe acutis, usque $7 \mathrm{~cm}$ longis; perianthio circ. $9 \mathrm{~cm}$ longo, tubo recurvato, angustato, circ. $5 \mathrm{~mm}$ lato, perigonii lobo supremo cucullato, circ. $3,2 \mathrm{~cm}$ lato; antheris linearibus circ. $18 \mathrm{~mm}$ longis.

Ober -Ka tanga: Élisabethville, décembre 1911 (Ad. Hock); Shisangwe, janvier 1912 (Homblé, no. 96).

Obs. - Diese Art scheint mit G. Quartinianus Rich. (cf. Baker in Fl. of trop. Afr., VII, p. 371) verwandt zu sein, ist aber verschieden durch die Behaarung der Blätter; sie ist auch mit G. Hockii nob. verwandt.

\section{Indigofera L. (Leguminosaceae).}

139. Indigofera Hockii De Wild. et Bak. fil., nov. spec.

Suffrutex humilis ad $I$. hilarem Ecklon et Zeyher valde accedens alis qua primo intuitu caulibus brevioribus calyce breviori et foliolis brevioribus facile distinguitur. 
Caules plures ex eadem radice orte $6-8 \mathrm{~cm}$ longi angulati. Stipulae filiformes. Folia 1-2-juga cum impari foliolis oblongis strigosopubescentibus saepius $5-7 \mathrm{~mm}$ longis et circ. $2 \mathrm{~mm}$ latis apice mucronatis petiolulis brevibus. Racemi pluriflori $15-25 \mathrm{~mm}$ longi rhachi canescenti-pubescente bracteis linearibus calyx circ. $4 \mathrm{~mm}$ longus dentibus angustis lineari-lanceolatis tuho pluries longioribus. Vexillum calyce longius extus dorso pubescens $6-7 \mathrm{~mm}$ longum. Alae carina conspicuae breviores. Carina naviculariformis circ. $7 \mathrm{~mm}$ longa apice pilis sparse obtecta. Ovarium lineari-oblongum pilis obtectum. Legumen ignotum.

Ober-Katanga: Élisabethville, 1911 (Ad. Hock).

Obs. - A close ally of I. hilaris Ecklon et Zeyher but the stems are shorter, the leaves are shorter and the calyx is shorter.

\title{
LXIX. Tialanchoe Adans. (Crassulaceae).
}

140. Kalanchoe Homblei De Wild., nov. spec.

Caulis erectus, ramosis, usque $1 \mathrm{~m}$ altus, ramis velutinis, pilis glandulosis, adultis subglabris; foliis medianis crassis, sessilibus, glabris, sicco papyraceis, obovatis vel ellipticis, margine dentato-undulatis, usque $13 \mathrm{~cm}$ longis et $5,5 \mathrm{~cm}$ latis; foliis superioribus reductis velutinis plerumque $1 \mathrm{~cm}$ circ. longis: inflorescentiis ramosis, ramis usque $20 \mathrm{~cm}$ longis, velutinis; cymis plus minus dichotomibus, rachide velutino, floribus bracteolatis, bracteolis linearibus, ciliatis, usque $3 \mathrm{~mm}$ longis; calyce inflato usque $5 \mathrm{~mm}$ longo, sparse velutino, ciliato; corolla lutea, tubo extus velutino, basi inflato usque $1 \mathrm{~cm}$ longo, lobis ellipticis, acuminatis usque $4 \mathrm{~mm}$ longis et $1.5-2,5 \mathrm{~mm}$ latis; ovario glabro, stylo brevi, capitato; seminibus numerosis.

Ober-Katanga: Élisabethville, mai 1912 (Homblé, no. 656).

Obs. - Verwandt mit $K$, glandulosa Hochst. und speziell mit $K$. glandulosa var. benguelensis Engler.

\section{L. Über die systematische Stellung von Gleditschia africana Welw.}

\author{
Von H. Harms.
}

(Originalarbeit.)

\section{Erythrophloeum africanum (Welw.) Harms.}

Syn.: Gleditschia africana Welw. ex Bentham in Trans. Linn. Soc. XXV (1865), 304; Oliv. Fl. Trop. Afr., II (1871), 265; Hiern, Catal. Afric. Pl. Welwitsch, I (1896), 289. - Caesalpiniodes africanum 0. Ktze. Rev. gen., I (1891), 167. - Erythrophloeum pubistamineum P. Hennings in Gartenflora XXXVIIL (1889), Heft 2, S. 41. Abbildg. 8; Schinz in Mém. Herb. Boiss., no. 1·(1900), 119 (var. parvifolium Schinz); Volkens, Nutzpflz. Togos, in Notizbl. Bot. Gart., App. XXII. no. 1 (1909), 11. 